\title{
Recent Advances in Design and Signal Processing for Antenna Arrays
}

\author{
Wei Liu, ${ }^{1}$ Des C. McLernon, ${ }^{2}$ Andy W. H. Khong, ${ }^{3}$ and Lei Yu ${ }^{4}$ \\ ${ }^{1}$ Department of Electronic and Electrical Engineering, University of Sheffield, Sheffield S1 3JD, UK \\ ${ }^{2}$ School of Electronic and Electrical Engineering, University of Leeds, Leeds LS2 9JT, UK \\ ${ }^{3}$ School of Electrical and Electronic Engineering, Nanyang Technological University, Singapore 639798 \\ ${ }^{4}$ School of Electronics and Information Engineering, Harbin Institute of Technology, Harbin 150001, China
}

Correspondence should be addressed to Wei Liu; w.liu@sheffield.ac.uk

Received 19 July 2016; Accepted 20 July 2016

Copyright (C) 2016 Wei Liu et al. This is an open access article distributed under the Creative Commons Attribution License, which permits unrestricted use, distribution, and reproduction in any medium, provided the original work is properly cited.

An array of antennas provides additional degrees of freedom in the spatial domain compared to a single antenna, which, with the aid of advanced signal processing algorithms, can be exploited for interference suppression/beamforming, direction of arrival estimation, target tracking, and localisation. It has a wide range of applications such as wireless communications, sensor networks, radar, satellite navigation, and biomedical engineering. In particular, with the extensive research activities devoted to the next generation wireless communication systems, antenna array design and signal processing have received unprecedented attention since the two underpinning 5G technologies, massive MIMO and millimetre wave communications, are all based on antenna array systems.

The aim of this special issue is to present the most recent advances in the area of design and signal processing for various antenna arrays and their applications. Both review articles and original contributions were invited from researchers working in this very important area and in total we received 32 submissions and 15 were accepted for publication.

The accepted papers cover a wide range of topics within the specified area of the special issue. Roughly speaking, they fall into the following six main topics. The first topic is antenna design for array applications, including two papers: "A SIW Antipodal Vivaldi Array Antenna Design" and "Wideband Dual-Element Antenna Array for MIMO Mobile Phone Applications"; the second topic is antenna array pattern synthesis or fixed beamformer design, including three papers: "Design of Wideband Multifunction
Antenna Array Based on Multiple Interleaved Subarrays," "Asymmetric Shaped-Pattern Synthesis for Planar Antenna Arrays," and "An Opportunistic Array Beamforming Technique Based on Binary Multiobjective Wind Driven Optimization Method"; the third topic is adaptive beamforming and interference suppression, including four papers: "Adaptive Array Beamforming Using a Chaotic Beamforming Algorithm," "Assessment of Measurement Distortions in GNSS Antenna Array Space-Time Processing," "Beam Tracking in Switched-Beam Antenna System for V2V Communication," and "Nonfeedback Distributed Beamforming Using Spatial-Temporal Extraction"; the fourth topic is direction/angle of arrival estimation, including three papers: "Two-Dimensional Direction-of-Arrivals Estimation Based on One-Dimensional Search Using Rank Deficiency Principle," "Transmit/Receive Spatial Smoothing with Improved Effective Array Aperture for Angle and Mutual Coupling Estimation in Bistatic MIMO Radar," and "ReducedDimension Noncircular-Capon Algorithm for DOA Estimation of Noncircular Signals"; the fifth topic is synthetic aperture related applications, including two papers: "Integration of Frequency Domain Wideband Antenna Nulling and Wavenumber Domain Image Formation for MultiChannel SAR" and "Tracking of Range and Azimuth for Continuous Imaging of Marine Target in Monopulse ISAR with Wideband Echoes"; the last topic is massive MIMO signal processing for next generation wireless communications, including one paper "Main-Branch Structure Iterative Detection Using Approximate Message Passing for Uplink Large-Scale Multiuser MIMO Systems." 


\section{Acknowledgments}

Finally, we would like to thank the authors for their valuable contribution to this special issue and the anonymous reviewers for their kind help and constructive comments, without which we would not have been able to complete this special issue with such a set of high-quality papers covering this wide range of topics.

Wei Liu

Des C. McLernon Andy W. H. Khong

Lei Yu 


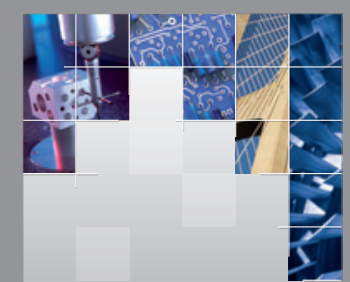

\section{Enfincering}
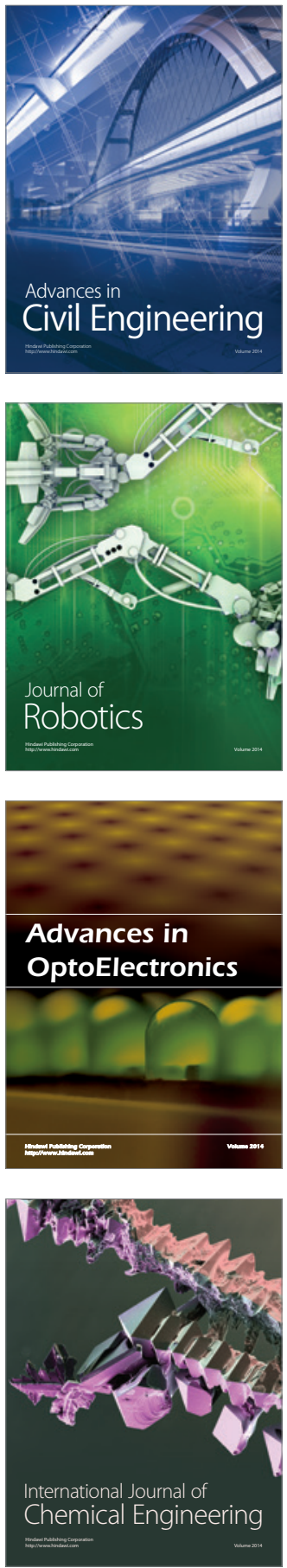

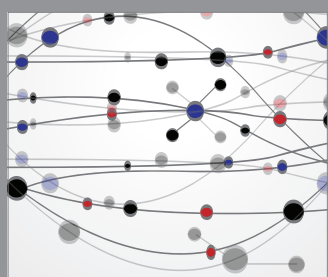

The Scientific World Journal

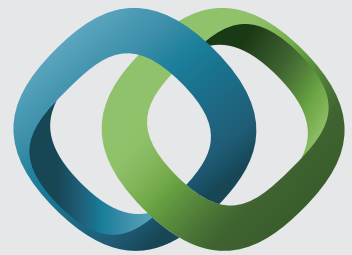

\section{Hindawi}

Submit your manuscripts at

http://www.hindawi.com
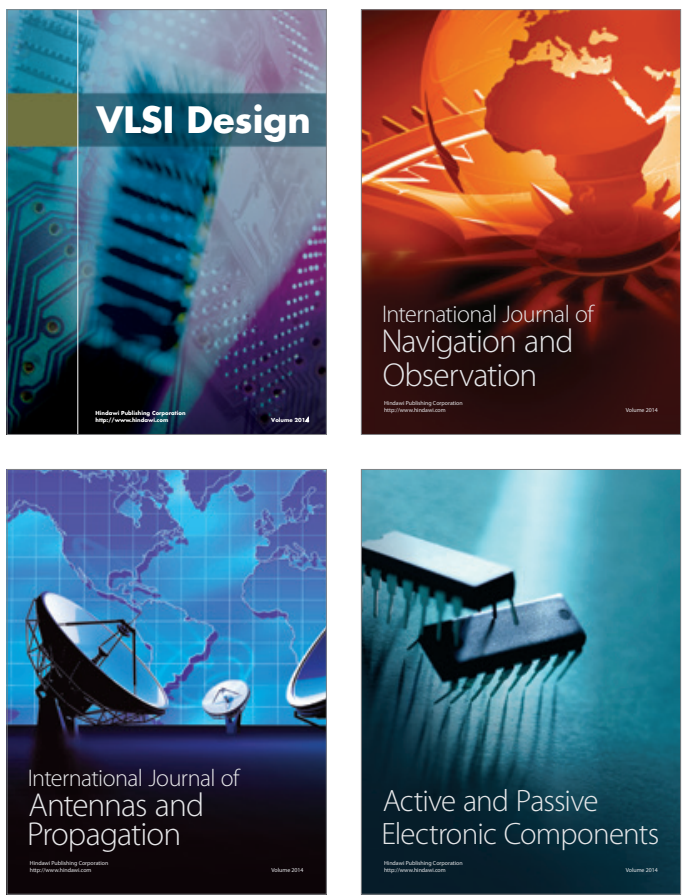
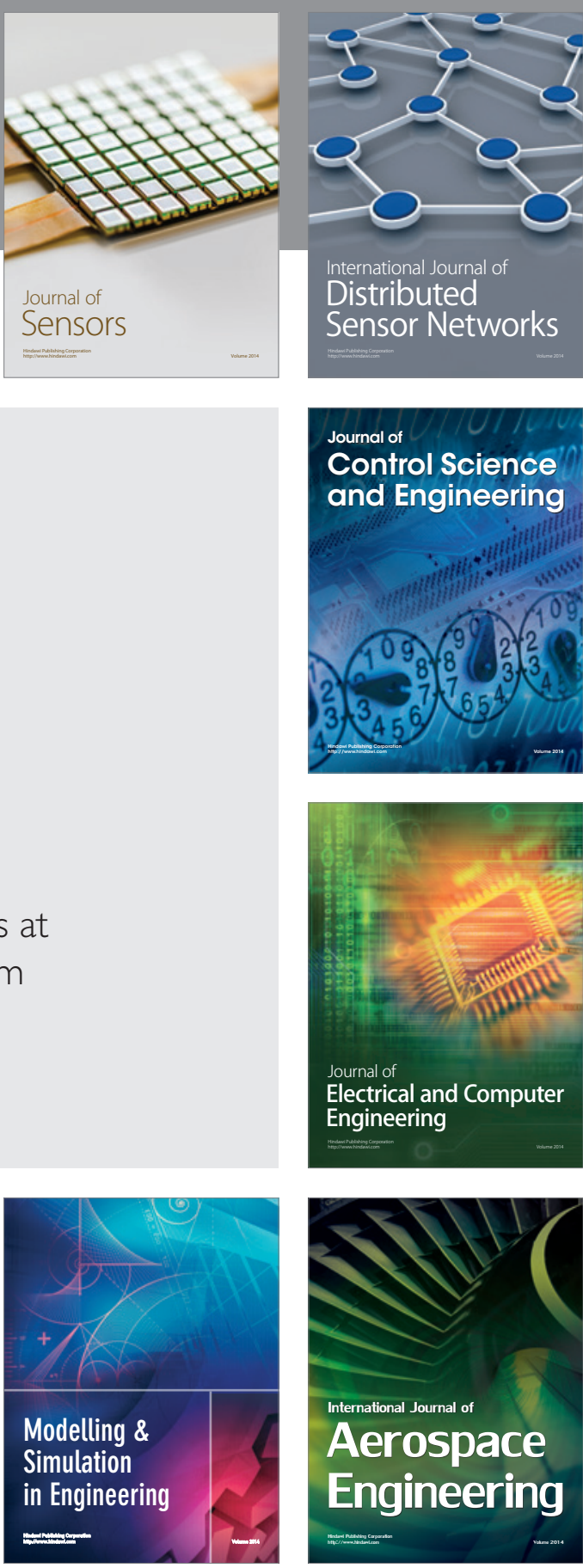

International Journal of

Distributed

Sensor Networks

Journal of

Control Science

and Engineering
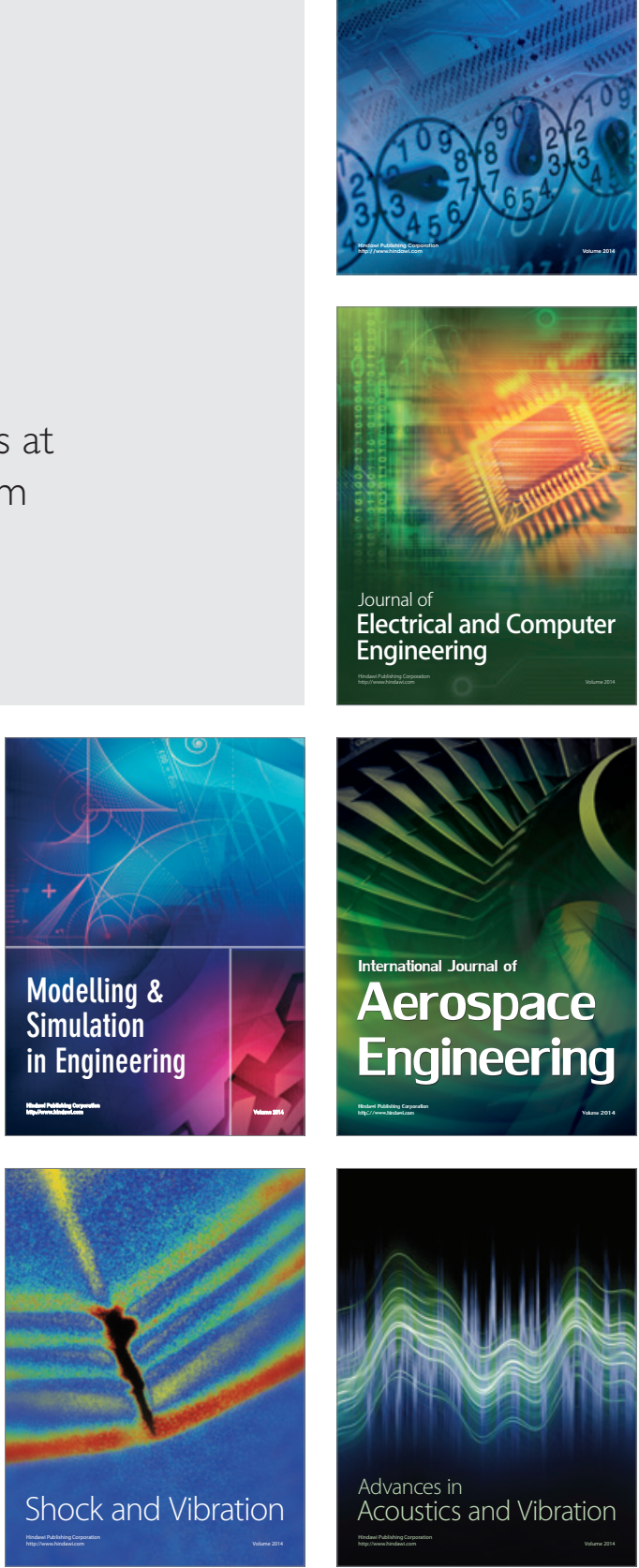